\title{
Hybrid Framework of, EWGM-FMEA, Analytical Hierarchy Process and Risk Balance Score Card for Risks Assessment in Energy Sector
}

\author{
Sahar Mohammad Al Mashaqbeh" ${ }^{1, ~ *, ~ J o s e ~ E d u a r d o ~ M u n i v e ~ H e r n a n d e z ~}{ }^{1}$, \\ Mohammad Khurshid Khan ${ }^{2}$ \\ ${ }^{1}$ Department of Mechanical and Automotive Engineering, University of Bradford, Bradford, UK \\ ${ }^{2}$ Department of Mechanical Engineering, Abdul Wali Khan University, Mardan, Pakistan \\ Email address: \\ s.m.a.almashaqbeh@bradford.ac.uk (S. M. AL Mashaqbeh) \\ *Corresponding author
}

\section{To cite this article:}

Sahar Mohammad Al Mashaqbeh, Jose Eduardo Munive Hernandez, Mohammad Khurshid Khan. Hybrid Framework of, EWGM-FMEA, Analytical Hierarchy Process and Risk Balance Score Card for Risks Assessment in Energy Sector. International Journal of Engineering Management. Vol. 2, No. 3, 2018, pp. 58-66. doi: 10.11648/j.ijem.20180203.12

Received: October 30, 2018; Accepted: November 21, 2018; Published: December 17, 2018

\begin{abstract}
Power plants are very important for continuous electricity energy supply and have been affected by many disruptions. Furthermore, the power grid is a critical item for both economy and society. Accordingly, the aim of this paper is to adopt a risk assessment tool combining an improved Failure Mode and Effect Analysis (FMEA), Analytical Hierarchy Process (AHP) and enhanced Risk Balance Score Card (RBSC) to model nine risk categories in the energy sector. The outputs of the improved FMEA methodology will be utilised as the inputs for the BSC-AHP framework. The improved FMEA methodology combines the exponential and weighted geometric mean to overcome some drawbacks of the conventional FMEA. The approach helps the top management in prioritising 84 risk indicators particularly, in power plants. The results of this model elucidate that the highest priority (most risky perspective) is for the supply chain perspective with $24.2 \%$ of the influence, followed by the internal and operational business process perspective with $18.4 \%$. In this perspective, the technical risk is the key risk with $10.4 \%$ followed by the disruption risk with $9.4 \%$ while the lowest priority risk in this perspective is the project neglect risk with $2.5 \%$. The sustainability perspective coming as the third priority perspective with $17.7 \%$, where the environmental and safety health category covers about $41.7 \%$, followed by the technological pillar with $35.5 \%$ and the social pillar with $22.8 \%$. At the fourth level, the customer/demand perspective is coming with $14 \%$, where the load forecasting risk has the highest priority in this perspective with $49 \%$. The learning and growth perspective stay at the fifth level with $13 \%$ where the human resources risks category has more influence than the management risks category. The lowest risk perspective priority is the economic perspective with $12.7 \%$. These results will help the top management in taking a holistic view of various non-technical risks at the strategic level and the priority for each one then, the suitable decision can be taken. The significance of this research is in presenting a novel improved for the traditional FMEA and combining it with the BSC-AHP methods to improve the risk assessment process of 84 risks of six perspectives of BSC in power plants at the strategic level.
\end{abstract}

Keywords: Exponential Weighed Geometric Mean (EWGM), FMEA, RPN, AHP and BSC

\section{Introduction}

The energy sector has been facing risks associated with economic, social, environmental, technological and a wide group of risks through the supply chain. Significant adverse effects in the energy sector in short-term and long-term will result from these risks and disruptions [1]. According to
Afgan et al [2], a fluctuation in the oil price has a major influence of sourcing and distribution strategy decisions, where the fuel accounts for around (1/4) to (1/3) of transport operating costs, therefore, any variation in the oil price has a direct impact on the supply costs of shippers. Moreover, Gonzalez (Gonzalez, 2015) illustrates that the effects of liberalisation process of energy markets industry are 
considerable; the competitive of the energy market is increased, which also changed the stochastic pricing energy approach. Furthermore, the energy market players have encountered the increasing risk level, therefore; the movements of the market will consider the keystone for the market player's decision-making process for managing controllable risks (market risk, credit risk, and operational risk) or for uncontrollable risks like the weather; which affect production and demand. Thus, powerful operational and planning decisions additionally, appropriate risk management strategies should be adopted and taken by the energy market players. Due to that, the development of risk model of electricity chain is an important need, to predict, address and manage these risks.

An improved FMEA methodology has been used to identify nine risk categories including a proper 84 Risk Indicators (RI's) within all these categories through the Lifecycle stages of power plants (explained in our conference papers). This methodology improves the traditional FMEA and overcomes some of the related drawbacks by combining the exponential and weighted geometric mean by considering the weights for the three risk factors ( severity, occurrence and detection) through utilising the AHP. The results of this improved methodology demonstrate that the duplication number of Risk Priority Number (RPN) have been decreased additionally, the results are more reasonable and depend on the three risk factors not just on the severity value. Afterwards, the outputs from the EWGM-FMEA methodology have been used as the inputs of the BSC-AHP to develop the related matrices accordingly, risks can be prioritised and weighted then, the most prominent risk indicator on power plants can be addressed.

This paper aims to establish a framework that combines the BSC-AHP tools with an improved FMEA to develop a framework to prioritise the risks in power plants. Therefore, the objectives of this research are to:

1. Identifying and understanding various types of risks (6 risks perspectives with 9 categories) in the energy sector;

2. Developing a risks framework that combines BSC and AHP to assess risks in the energy sector;

3. AHP technique helps in weighing and prioritising these different types of risks and overcomes the BSC drawback where the BSC help in categorising 84 risks.

To derive the risk priority values; a pairwise comparison of various risks has been conducted using the Expert choice software. These priorities have been used to determine the ranking level of each risks perspective and each risk.

\section{Literature Review}

To improve the service of generating electricity and minimise the risks, an integral approach for identification of the existing and the potential risks of power plants should be handled. Chan (Chan, 2009) shows that the potential risks along the operation of the business can disrupt the operation and cause significant losses, either these risks are catastrophic events like fire or flood or other smaller events like failures and breakdowns. All these risks will cause revenue losses, dropped production rates, inability to meet planned production goals, and these lead to reduce the reliability and hit the reputation of the company. In the same context, Garbuzova-schlifter and Madlener [5] clarify that risks present in each stage of life cycle, from the planning stage to the decommissioning stage of power plants and risks in one stage may affect other stages due to the integrity in the supply chain.

Risks should be understood by the companies to generate a plan for manage it furthermore, it is crucial to identify and understand the risks for minor and major risks to determine which failure will cause risk profiles particularly, man-made risks. Interestingly, the report describes seven key areas should be focused on it [6].

Risk management is one of the most relevant approaches and systematic application of strategies, procedures and practices management that have been introduced to identifying and analysing risks which exist through the whole life of product or process. The risk management needs in energy sector emerge from the role of power plants which is very crucial for continuous and reliable energy supply [4]. $\mathrm{Wu}$ and Olson [7] display that enterprise risk management is part of the strategic planning process Furthermore, enterprise risk is incorporated across the corporate strategy of an enterprise Risks are present in all stages, from the commission phase to decommission. According to [8] risks in one stage may affect other stages due to the integrity of the supply chain. Organisations share price is impacted by $7 \%$ from a significant supply chain disruptions either these disruptions are natural disasters, production issues, shortage of parts, recalls etc. [9]. Therefore, it is important to identify risk factors in all stages: commissioning and starting; fuel supply and delivering; operating, running, maintenance and Ash disposal; and finally the decommission stage). Thus, it is important to develop a comprehensive, coherent, methodological, structured and systematic approach to identify and assess the risks. In order to that, the risk mitigation plans can be developed and implemented.

AHP is one of the most frequently Multi-Criteria Decision Making Process (MCDM) methods used to solve complicated problems in various research areas. This refers to many reasons. Firstly, the natures of AHP, where it can decompose the complex decision problem into a hierarchical structure and execute a pairwise comparison between the criteria of a hierarchy. Secondly, other MCDM methods need certain many data but AHP needs less intensive data and can be applied with limited data. Thirdly, AHP can use quantitative and/or qualitative criteria. Fourthly, the preferences in AHP are obtained for two criteria at the same time but for other scale rates just one criterion at a time. Finally, AHP is capable of capturing the subjectivity and the objectivity and gives reasonable results of the decisionmaking process [5].

The complexity and interdependency of energy sector either in critical infrastructure or in key resource for today's 
society and economy life. This study focuses on the accident risks where the accident risk assessment is considered as the component in a holistic evaluation of energy security aspects and sustainability performance and they conclude that the second largest group of all man-made accidents worldwide accidents are located in the energy sector [10].

In the same area, Samvedi, Jain and Chan [11] assert that the risks of several energy technologies of society and environment that may happen not only during the actual energy generation but also at all phases of energy chains.

To estimate the likelihood of many risk events that may happen, the organisations can depend on the historical data, moreover the managers can use another tools and frameworks if the historical data are not available or insufficient to quantify risk exposure. In addition to that, he clarifies that the heat map score is used by managers to determine the priorities for risks. The risk events that score 15 or higher are the most likely and consequential and the strategy map offers a powerful framework for strategic and operational risks identifications [12].

As Radivojević and Gajović [13] confirm that the data related to the risks are not available thus, scholars depend on the experience and intuition of experts. In the same context, Geng et al [6] clarify that allocating and setting proper indicators to evaluate and assess the business performance is very important needs moreover, they represent that these indicators will be changed due to the nature of business operating. Furthermore, their study has been explained the obstacles that lie ahead in building effective and efficient indicators and are summarised as:

1. There is no detailed explanation or standardised process on collection, calculation and submission of data;

2. The indicator system is a voluntary one and may be chased with differing intentions.

Through developing an effective KRI's it should ensure that collecting and aggregating data have been done through elaborating all parties. In addition to that, they emphasise that the quality of the available data used for monitoring risks is a crucial element of developing the KRI's. Sources of information can help in choosing the KRI's [14]. Moreover, the availability of data can provide enrich information about the potential future risks. Internal data is unavailable for many risks, particularly those that have not been suffered previously. External risks expected to have a significant impact such as economic conditions changing, interest rate fluctuations, or new regulations and legislation. Organisations depend on the external data to develop the related KRI's where are roots cause and intermediate events, which can affect strategies, may emerge from outside sources of the organisations.

Based on the aforementioned, 84 risk indicators have been identified as shown in Table 1. These can impact the supply chain of generating electricity.

Table 1. Identified Risk Indicators.

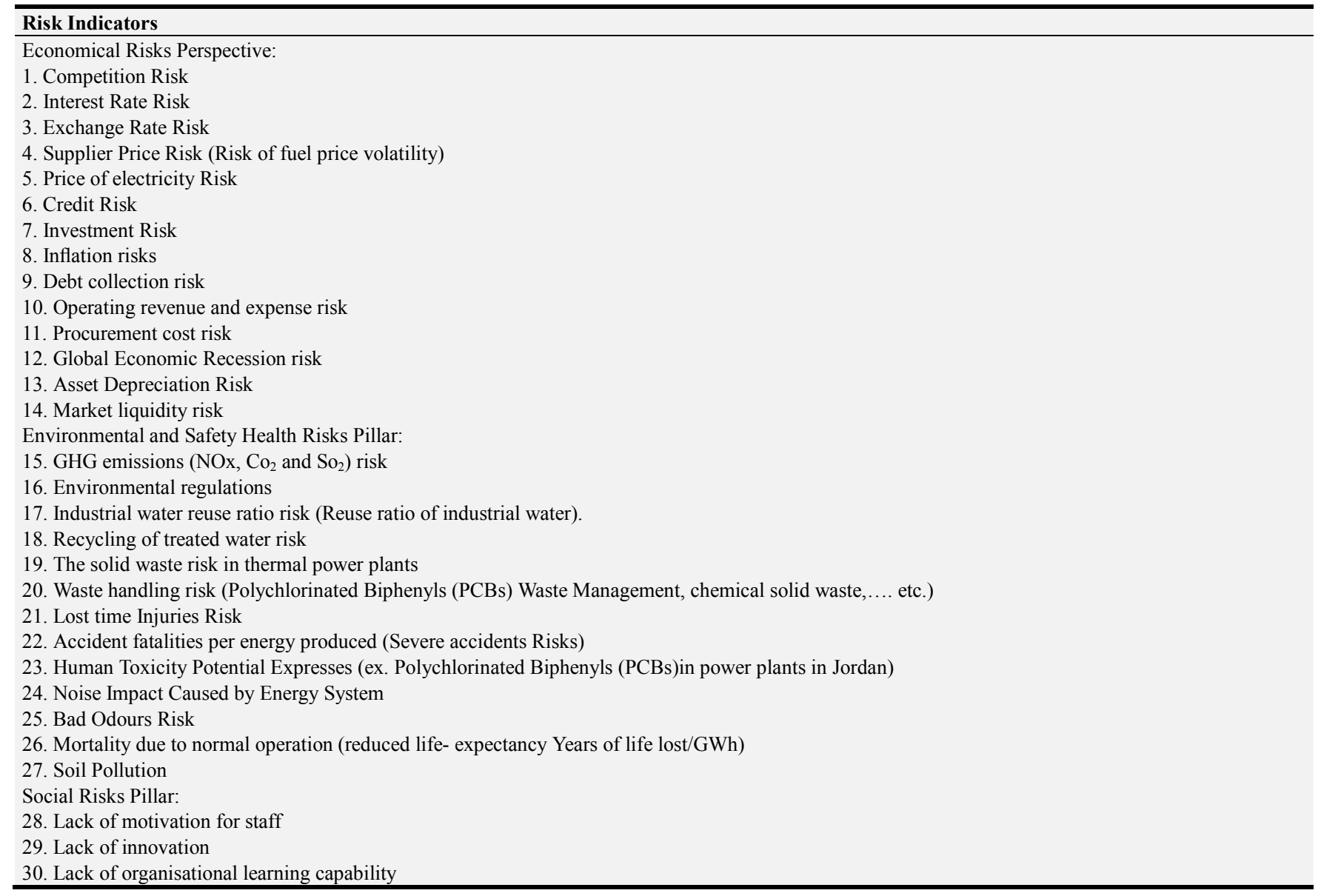




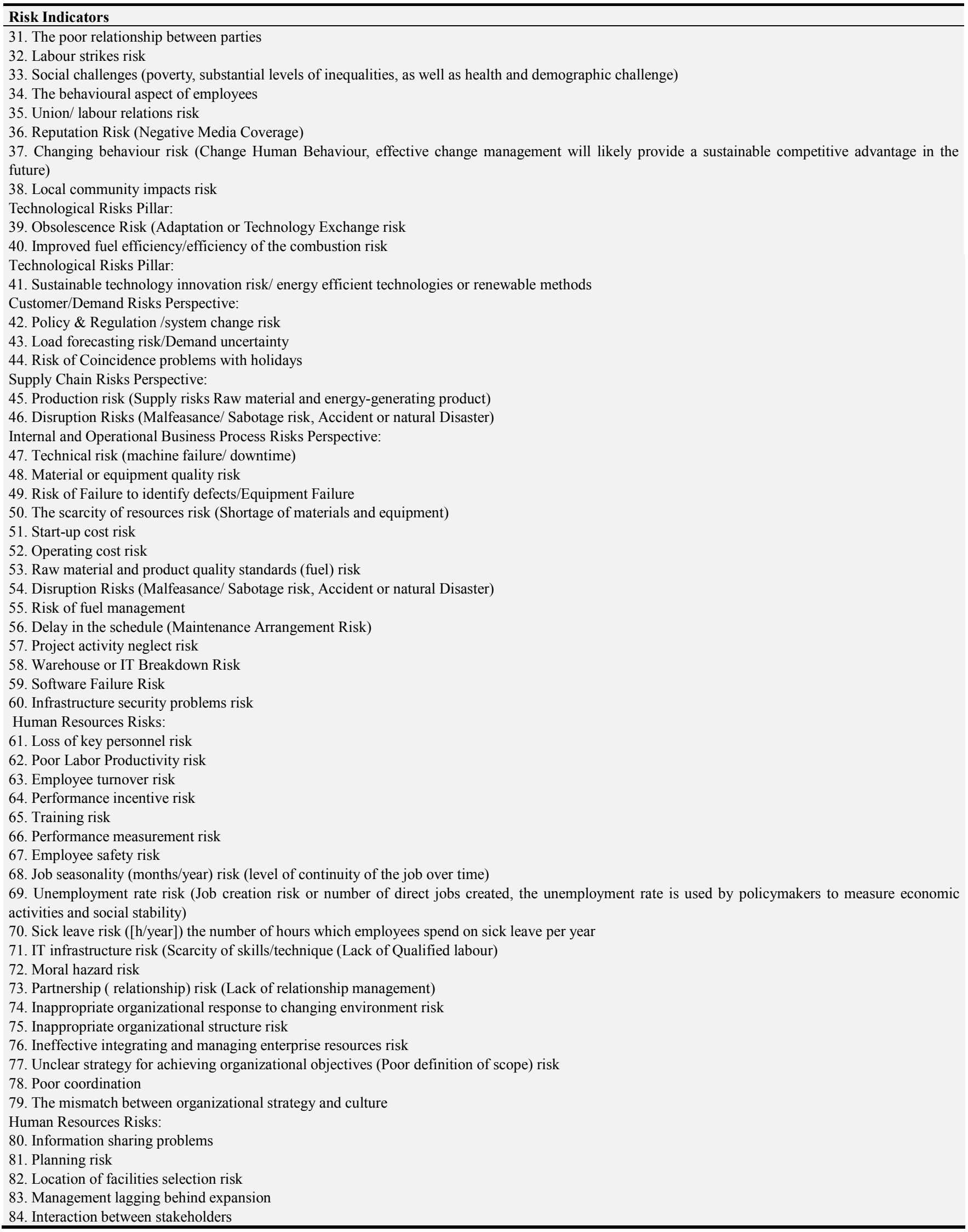




\section{Research Methodology}

Various risks have been collected from the literature and own experience (as identified in table 1), an improved FMEA is used to identify the risks ( explained in our conference paper), then the AHP has been applied to prioritise these risks depending on the output of the FMEA. The AHP is one of the MCDM processes that has been applied to synthesise expert judgments using the RPN values from the FMEA to show the effects of these risks on the performance of power plants. Based on the importance of each risk indicator, the comparison matrix will be generated afterwards, the priorities and the weights for all risks have been determined and categorised for three risk levels (High, medium and low-risk levels), Figure 1 represent these steps.

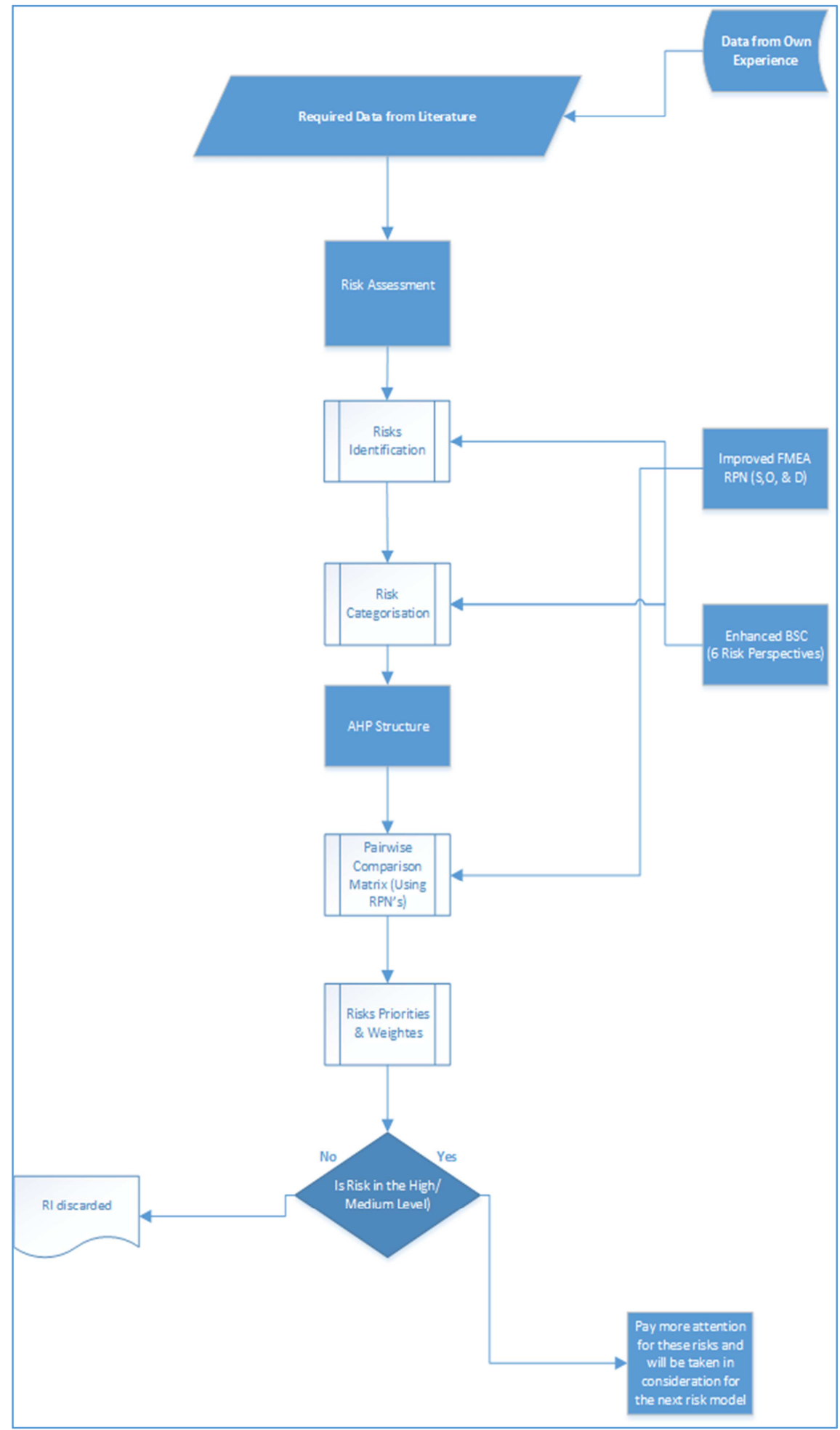

Figure 1. Research Methodology Steps. 
The analytical hierarchy process is a multi-criteria decision framework that allows constructing the decision into Goal, criteria, sub-criteria and alternatives. Its aim is to categorise this decision framework in a hierarchy and logical consequence.

\subsection{Modelling in AHP}

Depending on Table 1, a part of the hierarchy structure has been built as presented in figure 2 .

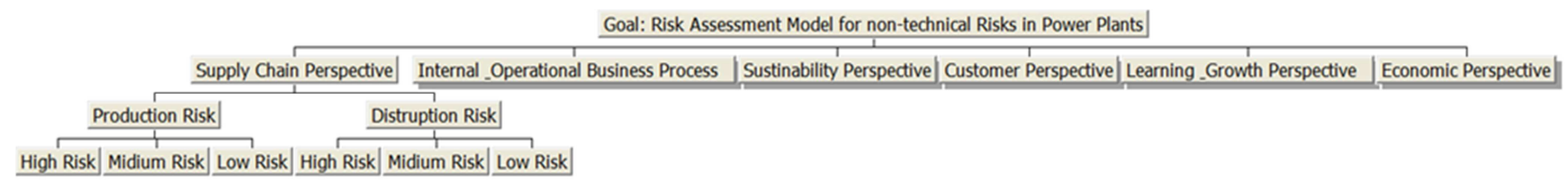

Figure 2. AHP Structure of the Risk Model.

The following process describes these hierarchy levels:

Level 0 Goal: Risk Assessment

Level 1 Main Criteria 1: Sustainability perspective:

Level 2 Sub-criterion 1.1: Environmental Pillar;

Level 3 Sub-Sub Criteria: 12 Risk Indicators (13 RI`s)

Level 3 Sub-criterion 1.2: Social Pillar;

Level 3 Sub-Sub Criteria: 12 Risk Indicators (11 RI`s)

Level 3 Sub-criterion 1.3: Technological Pillar (3 RI`s)

Level 1 Main Criteria 2: Economic perspective:

Level 2 Sub-criterion (14 RI's)

Level 1 Main Criteria 3: Learning and growth perspective:

Level 2 Sub-criterion 3.1: Human Resources Risk; Level 3

Sub-Sub Criteria: 12 Risk Indicators (12 RI`s) Level 2 Subcriterion 3.2: Management Risks Level 3 Sub-Sub Criteria: 12 Risk Indicators (12 RIss)

Level 1 Main Criteria 4: Internal \& operational business process perspective:

Level 2 Sub-criterion (14 RI's)

Level 1 Main Criteria 5: Customer perspective:

Level 2 Sub-criterion (3 RI's)

Level 1 Main Criteria 6: Supply chain perspective: Level 2 Sub-criterion (2 RI`s)

Level 4 Alternatives: Three Risk Levels (High, Medium and Low)

1. The matrix values and the importance of the elements have been assessed and evaluated according to the Saaty scale from 1 to 9 [16].

2. The global priorities have been calculated depend on the values of local priorities that have been obtained for each risk level.

3. The result is a relative importance of each element and has been ranked according to the weights of the risks. Depending on the relative importance of the risk perspectives, the impact on the overall risk level can be considered through three different alternatives (High, Medium and Low-risk levels).

4. Ranking of risk level indicates that the level of overall risk for the selected power plant chain and the risk indicators can be determined from the weighting of the priorities for each risk indicator.

The developed risk model will help the top management to take a wise strategic decision to reduce the overall risk where [11] demonstrate that a risk affecting the strategic level is much more risky than one affecting the operational level.

\subsection{Pairwise Comparison}

After the AHP structure has been developed, the pairwise comparison matrix for each sub-structure model is accomplished. The priorities of each risk perspective and each risk indicator have been calculated using the Expert Choice software. In the pairwise comparison matrix, a comparing between each risk indicator in pairs to represent the influence of each risk on the performance of power plants. There are two options for this pairwise comparison, one option compares the risk indicator with respect to the goal and the second option compare the risk indicator with respect to a specific risk perspective.

\section{Research Findings}

The pairwise comparisons matrix for each risk indicator within the related risk perspectives are determined, the weights for each perspective and each risk indicators have been illustrated in Table 2 .

Table 2. Summary of the Figure AHP weights for the Six Risk Perspectives.

\begin{tabular}{lllll}
\hline Risk Perspective & Perspective Priority & Risk Indicators & Category Priority & Sub-Risk Indicators \\
\hline Supply Chain & 0.242 & Production Risk & Priority \\
Perspective & & Disruption Risk & 0.500 \\
& & Technical Risk & 0.500 \\
& & Disruption Risk & 0.104 \\
& & Delay in Schedule & 0.094 \\
Internal \& Operation & 0.169 & Fuel Quality & \\
Business Process & & Operating Cost Risk & \\
& & Material or Equipment risk & \\
& & Risk of Fuel Management & \\
& & Risk of failure to identify defects & 0.091 \\
\end{tabular}




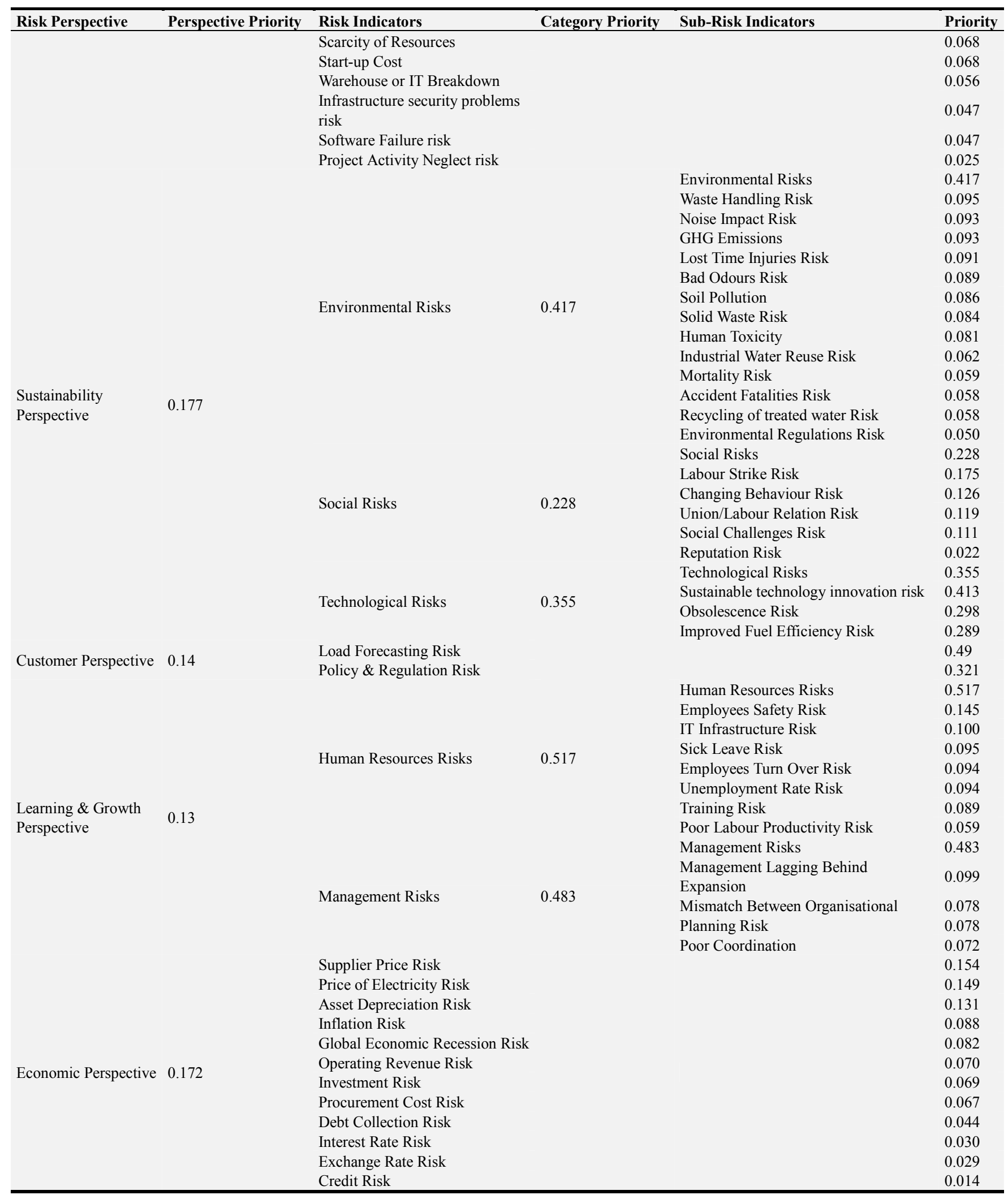

As shown in the priority table 2 and Figure 3; the highest priority (most risky perspective) is for the supply chain perspective, which includes two types of risks: (production risk and disruption risk) with $24.2 \%$ of the influence. Followed by the internal and operational business process perspective with $18.4 \%$ where the technical risk is the key risk in this perspective with $10.4 \%$ followed by the disruption risk with $9.4 \%$ and the lowest priority risk in this perspective is the project neglect risk with $2.5 \%$.

The sustainability perspective coming as the third priority perspective with $17.7 \%$, where the environmental and safety health category covers about $41.7 \%$, followed by the 
technological pillar with $35.5 \%$ and the social pillar with $22.8 \%$.

At the fourth level, the customer/demand perspective is coming with $14 \%$, where the load forecasting risk has the highest priority in this perspective with $49 \%$.

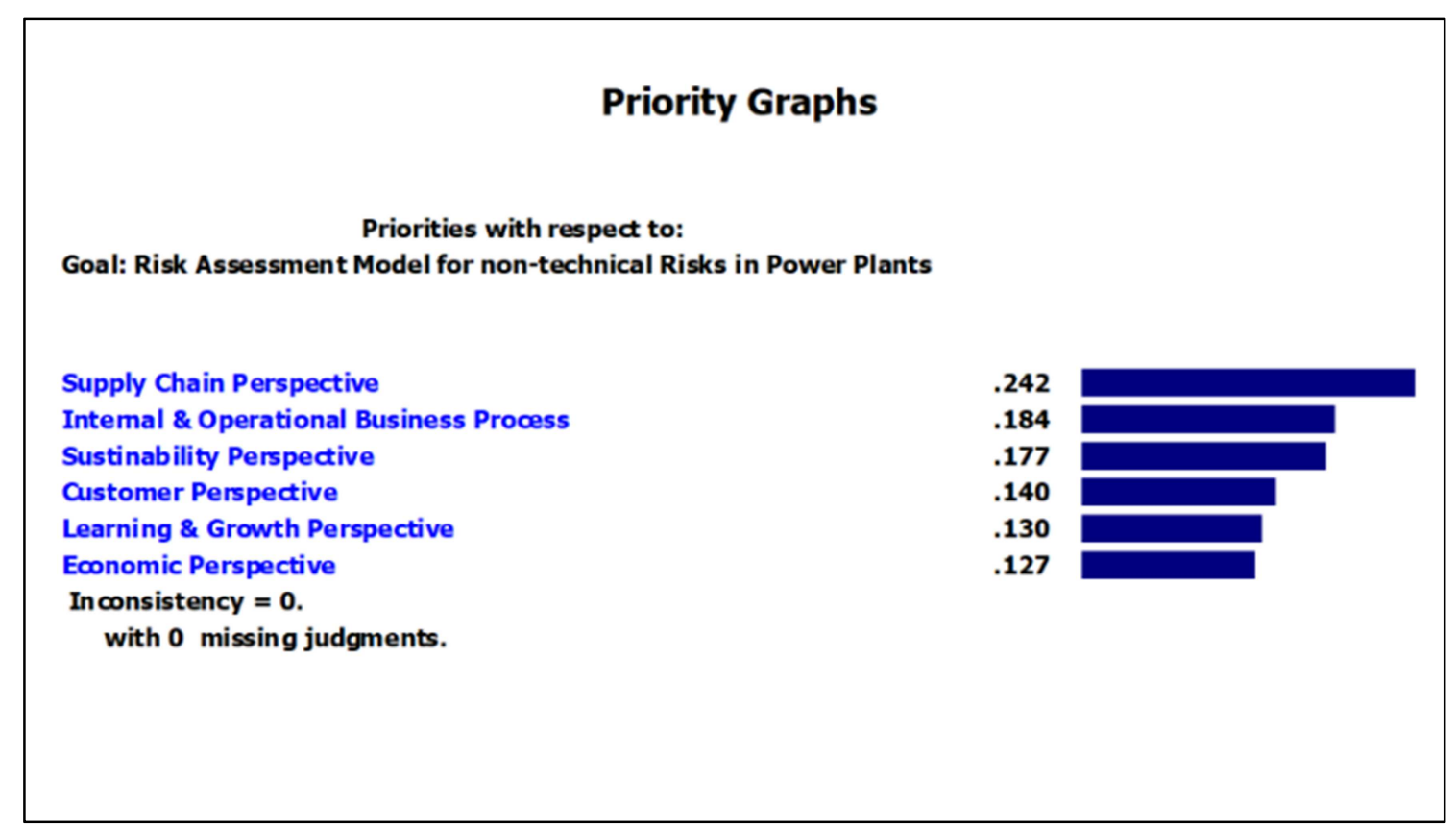

Figure 3. BSC-AHP Risk Results.

The learning and growth perspective stay at the fifth level with $13 \%$ where the human resources risks category has more influence than the management risks category. The lowest risk perspective priority is the economic perspective with $12.7 \%$.

In summary, the priority for each perspective and the level of the risk either in high, medium or in the low level have been elucidated in Table 3 .

As a whole, these results will be changed depending on power plant and the policy of the country. Therefore, the same FMEA methodology can be applied and different results can be generated.

Table 3. The Priorities for Six Perspective and the level of Risk.

\begin{tabular}{|c|c|c|c|c|c|}
\hline \multirow{2}{*}{ Risk Perspectives } & \multirow{2}{*}{ Traditional Method } & \multirow{2}{*}{$\begin{array}{l}\text { Modified } \\
\text { EWGMA }\end{array}$} & \multirow{2}{*}{ High-Risk Level } & \multirow{2}{*}{ Medium Risk Level } & \multirow{2}{*}{ Low-Risk Level } \\
\hline & & & & & \\
\hline Economic Perspective & 0.129 & 0.127 & 0.474 & 0.359 & 0.166 \\
\hline Sustainability Perspective & 0.171 & 0.177 & 0.463 & 0.303 & 0.234 \\
\hline Social Risks & 0.264 & 0.228 & 0.322 & 0.364 & 0.314 \\
\hline Environmental Risk & 0.487 & 0.417 & 0.595 & 0.213 & 0.192 \\
\hline Technological Risks & 0.249 & 0.350 & 0.399 & 0.369 & 0.232 \\
\hline Customer/ Demand Perspective & 0.169 & 0.140 & 0.43 & 0.381 & 0.189 \\
\hline Internal and Operational Perspective & 0.174 & 0.184 & 0.489 & 0.403 & 0.108 \\
\hline Supply Chain Perspective & 0.236 & 0.242 & 0.766 & 0.191 & 0.043 \\
\hline Learning \& Growth Perspective & 0.121 & 0.130 & 0.382 & 0.469 & 0.149 \\
\hline Human Resources Risks & 0.469 & 0.517 & 0.335 & 0.512 & 0.154 \\
\hline Management Risks & 0.531 & 0.483 & 0.432 & 0.424 & 0.144 \\
\hline
\end{tabular}

\section{Conclusions}

In this paper, a hybrid tool has been used to assess 84 risks in power plants. The BSC as a performance measurement tool has been enhanced and developed as a risk measurement tool with six perspectives rather than four and combined later with one of the MCDM to prioritise 84 risk indicators in power plants. These risks have been selected from literature and own experience. The comparison matrices have been conducted depending on the value of an improved FMEA RPN 's.

The developed models were applied for various 84 risks to determine the level of these risks either in the high, medium or low-level area afterwards, the Key risk indicators (KRI's) which have been located in the high and medium level area will be selected and the low-level risk will be discarded.

The results of this AHP model will be used to build the system dynamic model with nine sub-risk models. As shown, the most risk perspective is the supply chain perspective with $24.2 \%$ of the influence. Followed by the internal and operational business process perspective with $18.4 \%$. Depending on the priorities of all risk indicators, the results show that the top management should pay attention to various types of risks either supply chain risks, internal and operational risks or sustainability 
risks. The developed risk model will help the top management to prioritising the risks and support their decision making process.

\section{References}

[1] K. O. Achebe, "Risk Based Models for the Optimization of Oil and Gas Supply Chain Critical Infrastructure," 2011.

[2] N. H. Afgan, M. G. Carvalho, and N. V. Hovanov, "Energy system assessment with sustainability indicators," Energy Policy, vol. 28, no. 9, pp. 603-612, 2000.

[3] J. Gonzalez, "Modelling and Controlling Risk in Energy Systems," University of Manchester, 2015.

[4] S. T. Chan, "IDENTIFYING RISK FACTORS IN THE GENERATING SECTION OF THE POWER PLANTS By," no. May, 2009.

[5] M. Garbuzova-schlifter and R. Madlener, "AHP-based risk analysis of energy performance contracting projects in Russia," Energy Policy, vol. 97, pp. 559-581, 2016.

[6] Y. Geng, J. Fu, J. Sarkis, and B. Xue, "Towards a national circular economy indicator system in China: An evaluation and critical analysis," J. Clean. Prod., vol. 23, no. 1, pp. 216224, 2012.

[7] D. D. Wu and D. L. Olson, "Introduction to the special section on 'optimizing risk management: Methods and tools," Hum. Ecol. Risk Assess., vol. 15, no. 2, pp. 220-226, 2009.
[8] X. Liu and T. Arthanari, "A system dynamics model for managing corruption risks in dairy supply chains," 34th Int. Conf. Syst. Dyn. Soc., pp. 1-17, 2016.

[9] WEF, "Building Resilience in Supply Chain," Geneva, 2013.

[10] P. Burgherr, P. Eckle, and S. Hirschberg, "Comparative assessment of severe accident risks in the coal, oil and natural gas chains," Reliab. Eng. Syst. Saf., vol. 105, pp. 97-103, 2012.

[11] A. Samvedi, V. Jain, and F. T. S. Chan, "Quantifying risks in a supply chain through integration of fuzzy AHP and fuzzy TOPSIS," Int. J. Prod. Res., vol. 51, no. 8, pp. 2433-2442, 2013.

[12] B. R. S. Kaplan, "Risk Management and the Strategy Execution System," 2009.

[13] G. Radivojević and V. Gajović, "Supply chain risk modeling by AHP and Fuzzy AHP methods," J. Risk Res., vol. 17, no. 3, pp. 337-352, 2014.

[14] M. S. Beasley, B. C. Branson, B. V Hancock, and C. Landes, "Developing key risk indicators to strengthen enterprise risk management," 2010.

[15] M. A. Brown, D. D. Arcy, L. Melissa, Sharma Isha, and Li Yufei, Solid Waste from the Operation and Decommissioning of Power Plants. 2017.

[16] T. L. Saaty, "Decision making with the analytic hierarchy process," Int. J. Serv. Sci., vol. 1, no. 1, p. 83, 2008. 